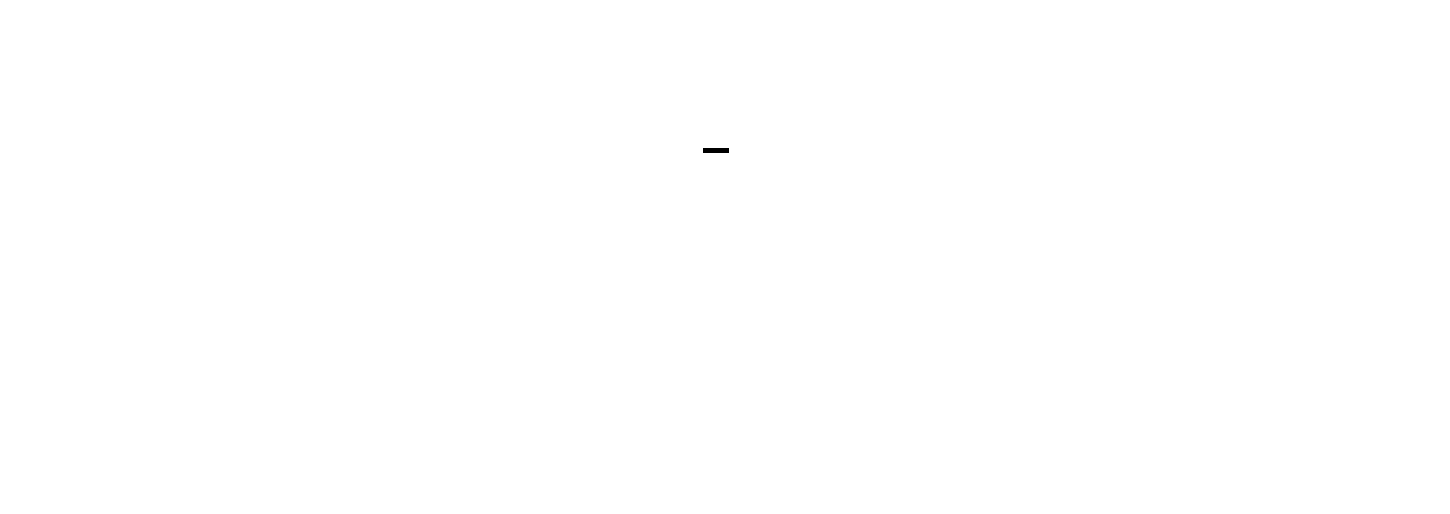

(تاريخ الاستلام 2004/7/7; تاريخ القبول 2005/4/25)

\title{
الملخص
}

أضف البولي أثلين بنسب مختلفة إلى مزائج الأسفلت - كبريت الحاوية على ذ علب مقفاوة ـة م ن

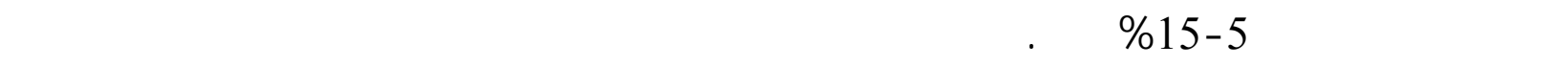

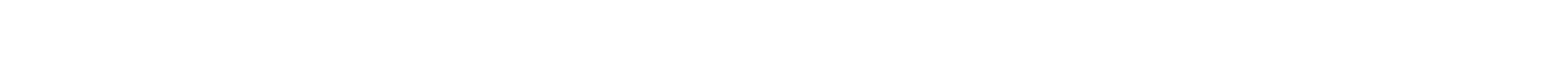

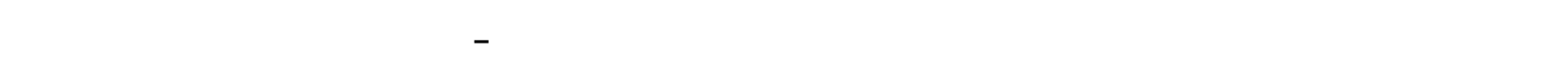

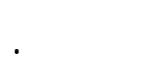

\section{Effect of poly ethylene addition on thermodynamic stability of Asphalt - Sulphur blends}

\author{
Imad A. AL- Dobouni \\ Lubna A.Salih \\ Department of Chemistry \\ College of Science \\ Mosul University \\ Shaymaa S. AL- Akidie
}

\section{ABSTRACT}

Poly ethylene was added in different percentages to Asphalt-Sulphur blends that contain from $5-15 \%$ by weight Sulphur. Physical properties of Asphalt - Sulphur - Poly ethylene blends were determined. The effect of poly ethylene addition on the homogeneity of Asphalt - Sulphur blends was studied after an aging period of more than one year at ambient temperatures.

\section{المقدمة}

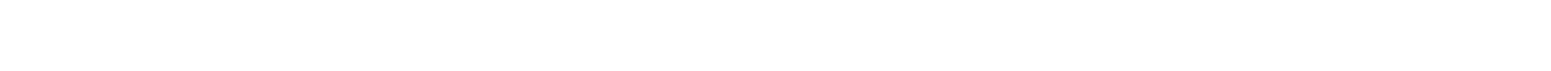
الماضي ظارَ لوفرته في المصادر الطبيعية، ولأسعاره المقبولة، ولما يمكن أن يضفيه من صفلت جي ــة 


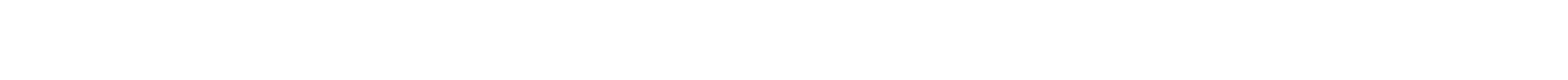
مفذاً للاستغلال الفضلات الكبريتية (Saylak \& Gallawy.,1975).

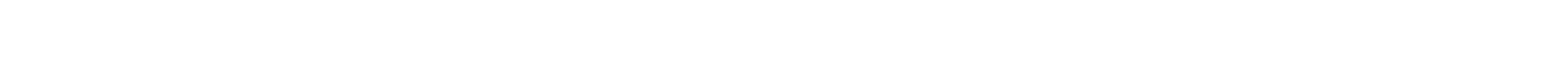

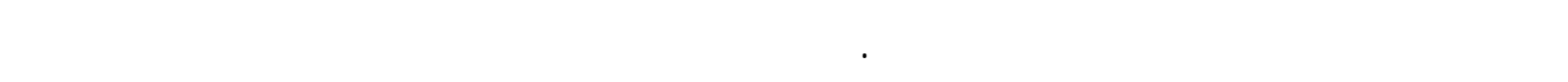

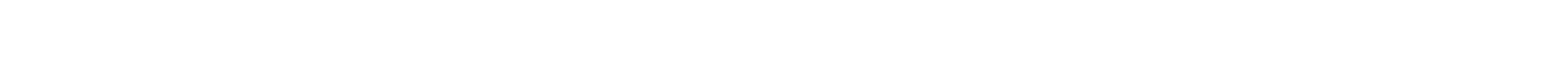
.(Rahimian,1976), (Quarles et al.,1965) ماسنركز عليه في هذه الدرلسة هو الأسلوب الأول، والذي يعتمد على مزج وإذابة الكبري ـت ف مي

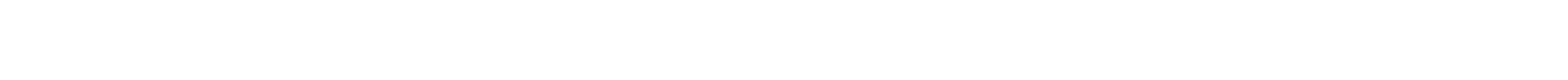
يظلب معدات متخصصة لأغراض المعالجة الكيميائية.

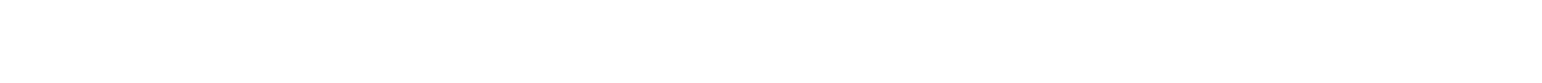

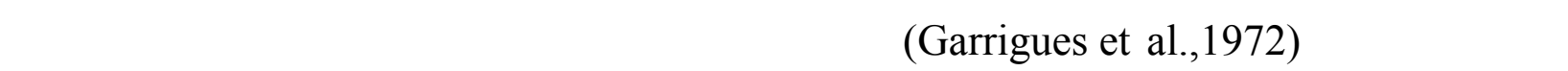
المستحصلة من الكبريت العنصري الحر إلى الأسفلت.

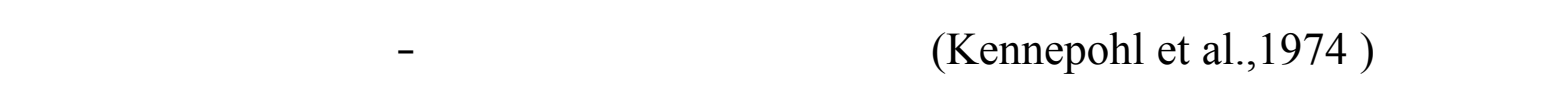

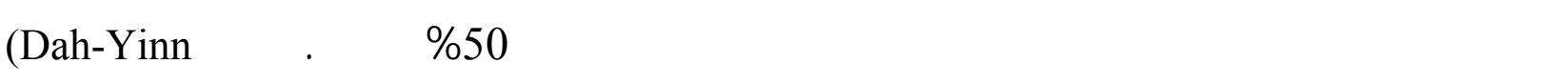
المانس Lee,1975)

الأسفلتين ونسب ثالي كبريتيد الهيدروجين المتحررمن المعالجة. كذلك ق لم (Sullivan et al.,1975)

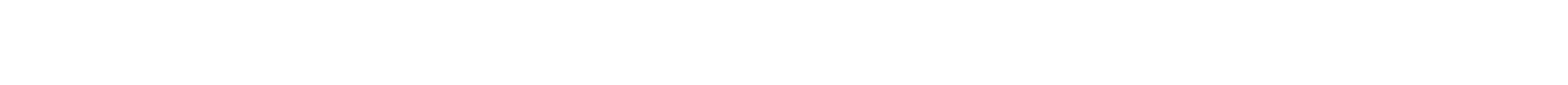

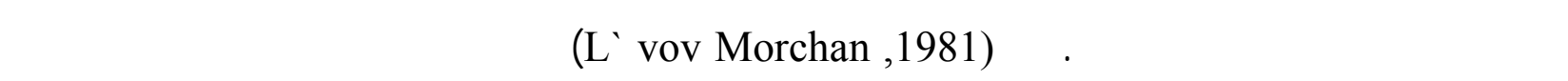
المضافة على مواصفلت المزيج المستخدم في التبليط. كذلك قلم (Fromm et al.,1981) بدرلسة ت أثير

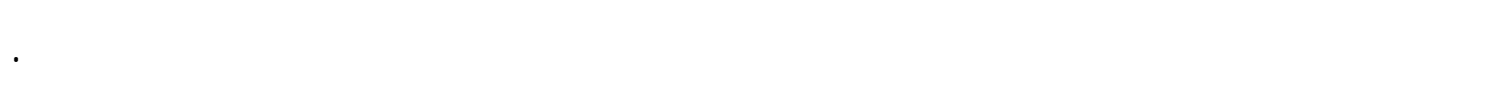

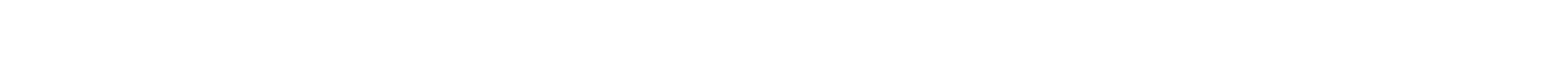

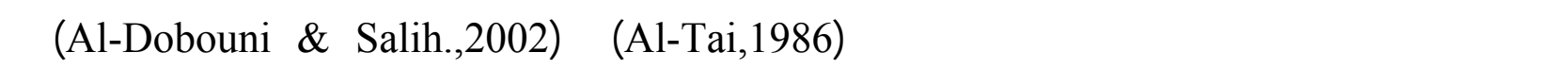
.(Samarai,1978)

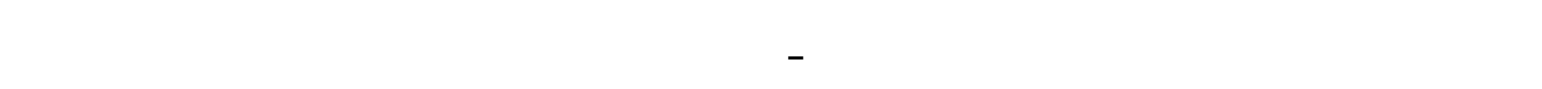
ثبوتيتها الثرموداينلميكية مما يؤدي إلى حدوث ظاهاهرة الفصل الحالات (Phase Separation) في المزيج النانج بعد فترة زمنية محدودة مما يؤدي إلى تدهور المواصفلت الفيزيائية لمزيج الأسفل -كبريت.

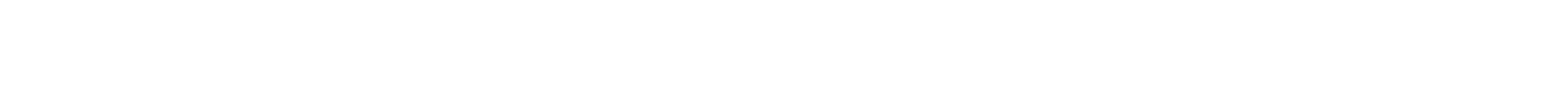

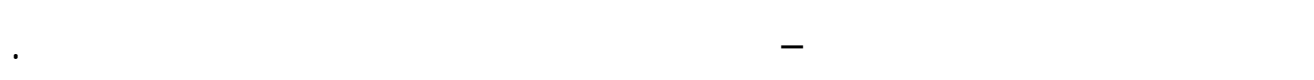




\section{الجزء المملي \\ أ -المواد المستهماة المبري}

1 -الأسفلت: لستخدم في هذه الدرلسة الأسفلت المنتج في مصف بيجي والذي يتصف بوزن أه ـوعي مق داره

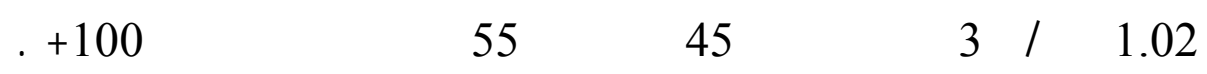

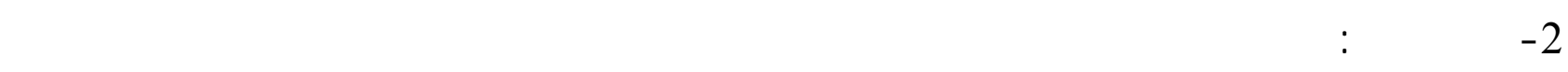
أثلين.

\section{ب -تحوبر الأسفلت بالبولي أثلالن}

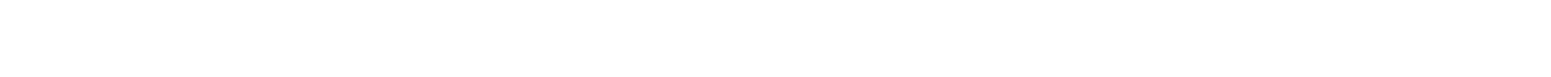
بوضع وزن معلوم من الأسفلت في دورق ثلاثي العنق مجهز بمحرك ميكانيكي ومحرار، مـ يضف إلف إل ق

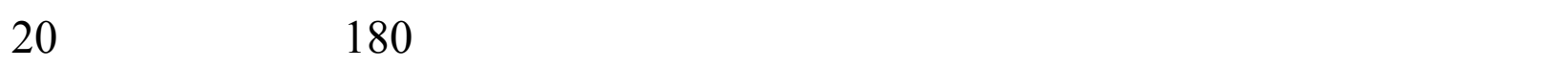

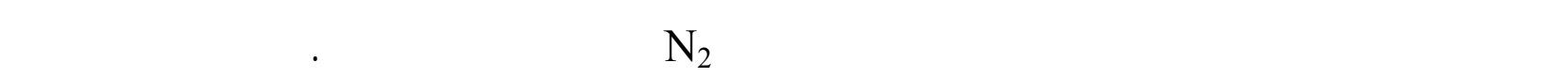

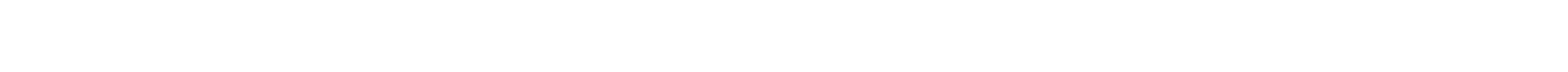

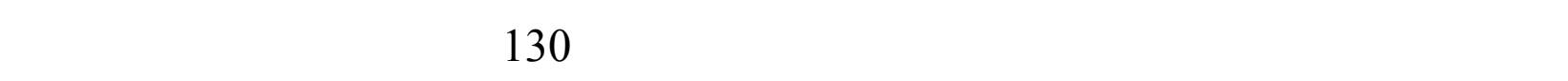

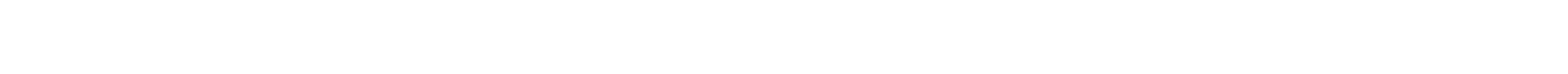

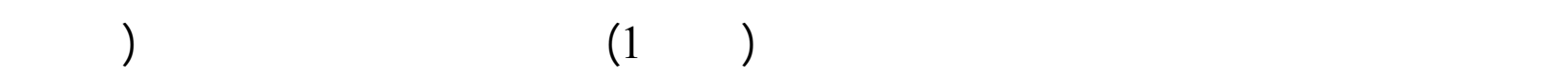

الجدول 1 : المواصفلت الفيزيائية للأسفلت بيجي المحور بيلساليب المزج الفيزيائي مع الكبري ـت أوم \& البولي أثلين.

\begin{tabular}{|c|c|c|c|c|c|c|c|c|c|}
\hline \multicolumn{5}{|c|}{ لسفلت بيجي المحور بالمزج الفيزيائي مع البولي اثلين } & \multicolumn{5}{|c|}{ لسفلت بيجي المحور بالمزج الفيزيائي مع الكبريت } \\
\hline \multicolumn{4}{|c|}{ بولي الثلين } & \multirow{2}{*}{ الصقلة } & \multicolumn{3}{|c|}{ كبريت } & \multirow{2}{*}{ الألموذج } & \multirow[t]{2}{*}{ الصفة المقلسة } \\
\hline$\% 8$ & $\% 4$ & $\% 2$ & $\% 1$ & & $\% 15$ & $\% 10$ & $\% 5$ & & \\
\hline 8 & 7 & 6 & 5 & رقو المولثرجيحة & 4 & 3 & 2 & 1 & أو الثم النموذج \\
\hline 53 & 51 & 47 & 46.8 & درجةالليونة & 47 & 43 & 47 & 45 & درجة الليونة \\
\hline 53 & 74 & 104 & 104 & الفانية & +65 & +100 & 91 & 55 & الفاذية \\
\hline 15.5 & 40 & 73 & +100 & الاسطظالة & +100 & +100 & +100 & +100 & الإسطالة \\
\hline-033 & 0.04 & -0.04 & -0.1 & دليل الفانية & -1.37 & -2.03 & -0.46 & -2.32 & دلل الفانية \\
\hline & & & & التجال ز زي الة & & & & & التجانس _ـه زي _ـاة \\
\hline
\end{tabular}


الجدول 2: الصفلت الفيزيائية للنماذج الأسفلتية المحورة بالبولي أثلنين والكبريت.

\begin{tabular}{|c|c|c|c|c|c|c|c|c|c|c|c|c|}
\hline \multicolumn{3}{|c|}{ 8\% وزن بولي الثلن } & \multicolumn{3}{|c|}{ 4\% وزن بولي الثلين } & \multicolumn{3}{|c|}{ 2\% وزن بولي اثلين } & \multicolumn{3}{|c|}{ \% وزن بولي الثلين } & \multirow{3}{*}{ الصفلة الصة } \\
\hline \multicolumn{3}{|c|}{ غسبة الكبريت } & \multicolumn{3}{|c|}{ نسبة الكبريت } & \multicolumn{3}{|c|}{ نسبة الكبريت } & \multicolumn{3}{|c|}{ نسبة الكبريت } & \\
\hline$\% 15$ & $\% 10$ & $\% 5$ & $\% 15$ & $\% 10$ & $\% 5$ & $\% 15$ & $\% 10$ & $\% 5$ & $\% 15$ & $\% 10$ & $\% 5$ & \\
\hline 20 & 19 & 18 & 17 & 16 & 15 & 14 & 13 & 12 & 11 & 10 & 9 & رقم النموذج \\
\hline 56 & 45 & 50 & 49 & 43 & 46.5 & 41.0 & 41.5 & 48.5 & 46.2 & 42 & 43 & الليونة \\
\hline 76 & 39 & 73 & 75 & 108 & 111 & 151 & 173 & 107.0 & 38 & 86 & 124 & الفانية \\
\hline 9 & 8 & 7 & 25 & 40 & 61 & 54 & 72 & 68 & 85 & 90 & +100 & الالسطالة \\
\hline 1.32 & -0.85 & -0.02 & 0.44 & 1.2 & 0.03 & -0.8 & -0.4 & 0.21 & 0.72 & -2.20 & -0.70 & دللل الفانية \\
\hline
\end{tabular}

\section{ج تتحوبر الأسفل بالكبربت}

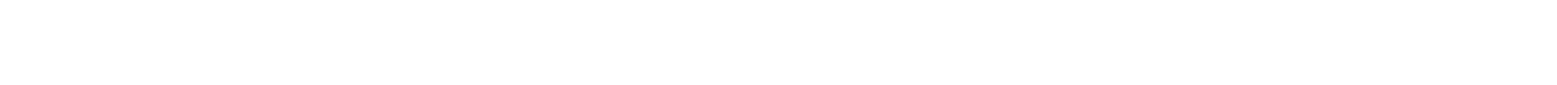

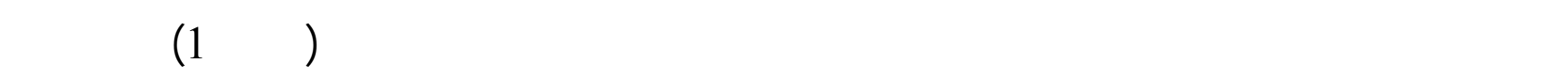

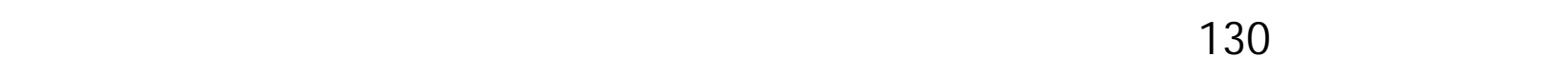
المختلة.

\section{د -تحسيد الموالهفلت الفيزبائية لنماذج الأسفل}

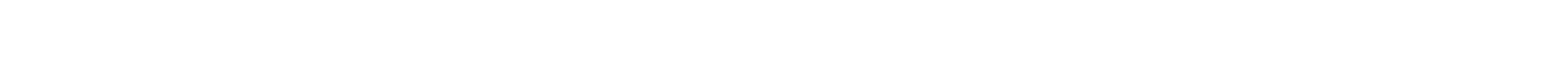

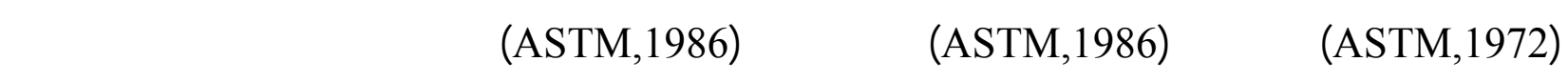
القيلسية المعتمدة عالمياً.

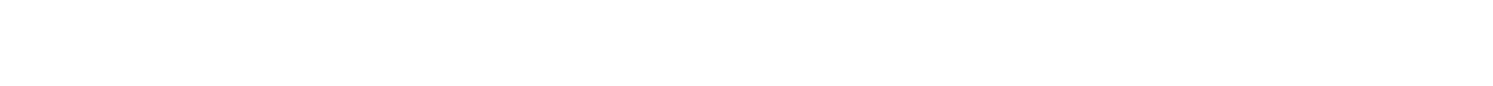

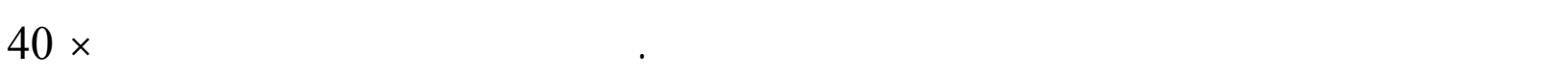

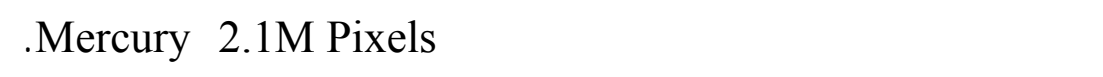

\section{النتائج والمنالثة}

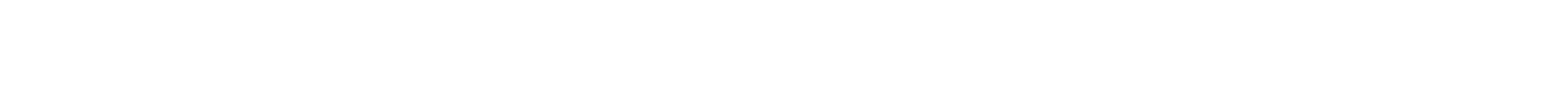

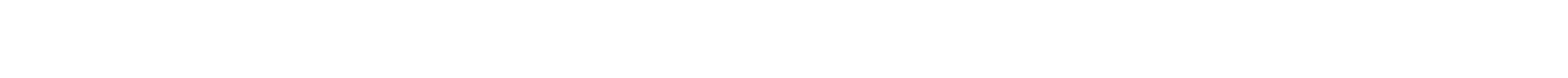

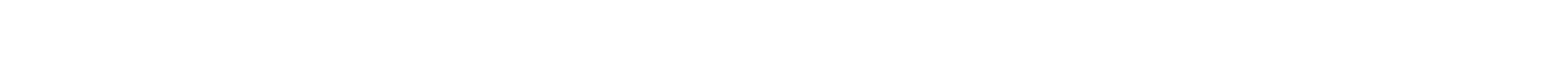

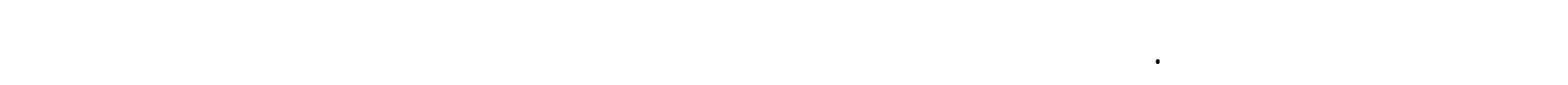

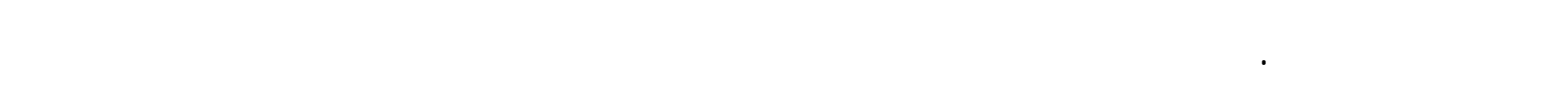

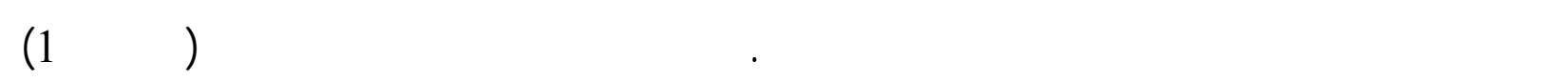

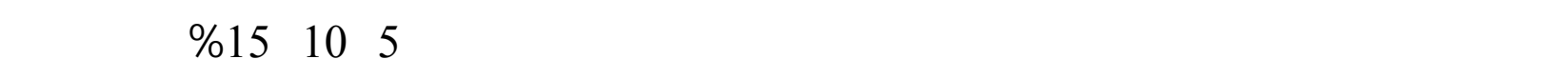




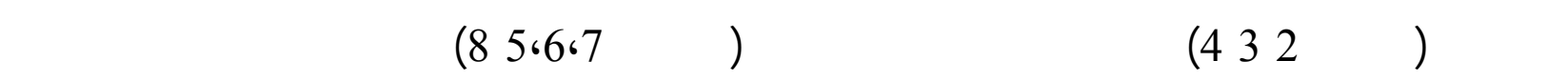

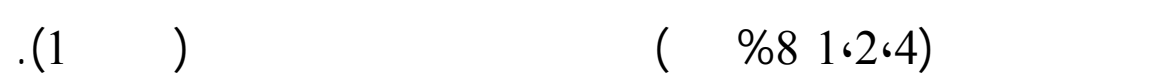

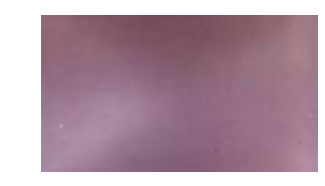

$$
\text { لأسفلت بيجي الاعتيادي }
$$

نماذج لُففلت بيجي المحورة بالبولي
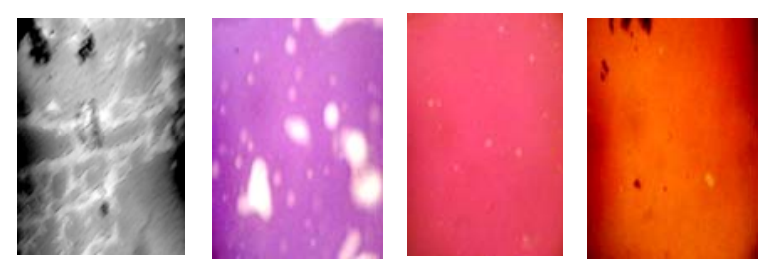

1\%بولي الثلين 2\%بولي الثلين 4\%بولي الثلين \% بولي انثلين
نماذج ألسفلت بيجي المحورة

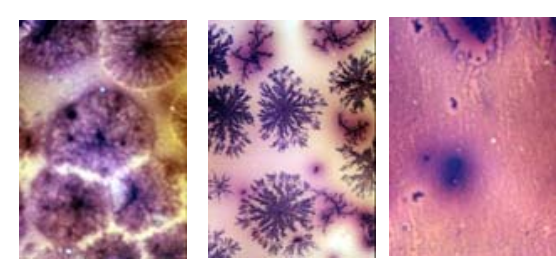

5\%كبريت 10\%كبريت 15\%كبريت

الشكل 1: الثررائح الميكروسكوبية لنماذج الأسفلت المحورة بالكبريت أو بولنطة البولي أثلين.

كذلك توضح لنا الصور الميكروسكوبية (للنماذج 4،3،2،سب فثل كلفة المحاولات والدرلس ـات

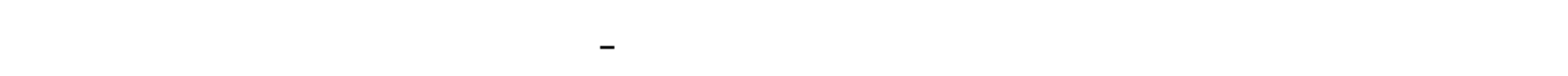

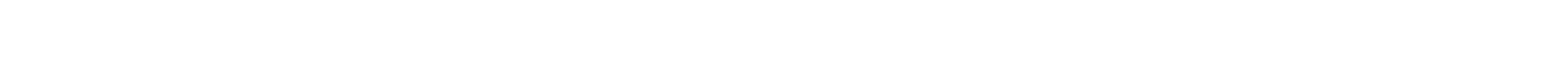
للظلم الأسفلتي. من ناحية لخرى، نجد أن إضافة كميلت مختلفة من البولي أثلين إلى الأسفلت قادت أيضا إلى تكوين

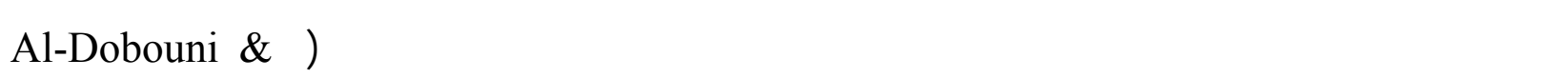

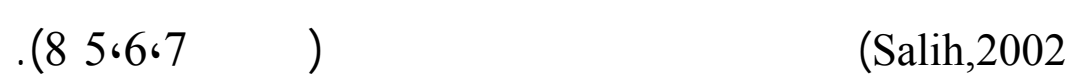

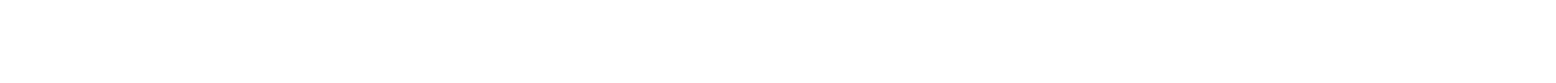

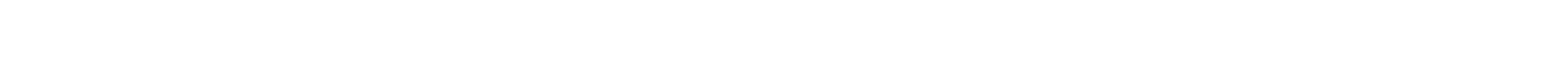
يؤدي إلى زيادة قابلية ذوبلن والنشار المادة الأخرى في الوبط الأسفلتي.

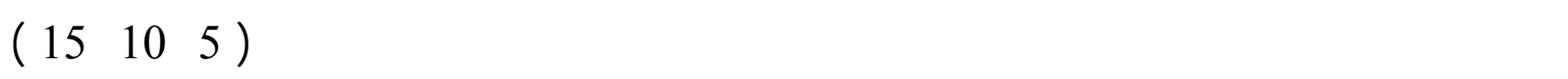

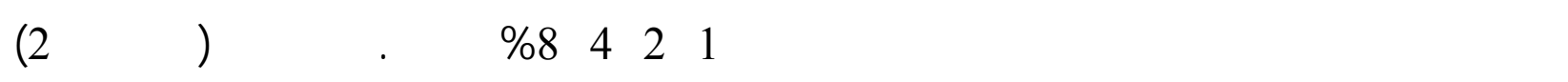

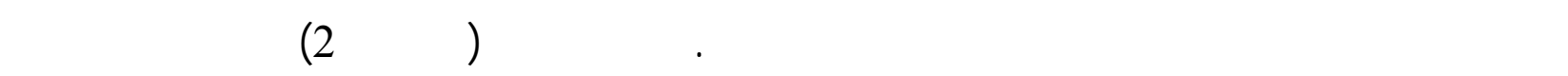

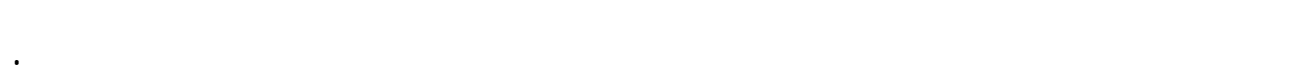


عند ملإظةطبيعة التغير في الصفلت الفيزيائية المتمثلة بدرجلت الليوذة والفاني ة واللس شطالة لنماذج الأسفلت نجد أن نهط التغيير لم يختلف بشكل علم عن النط الذي لأظه بقية البلحثين الذن قلموا

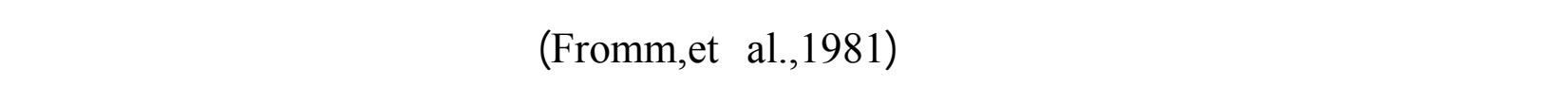

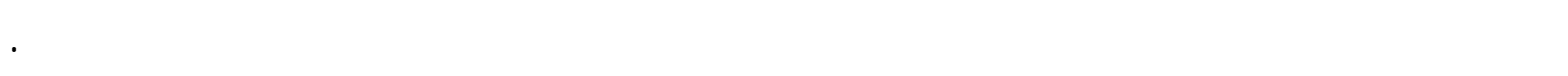

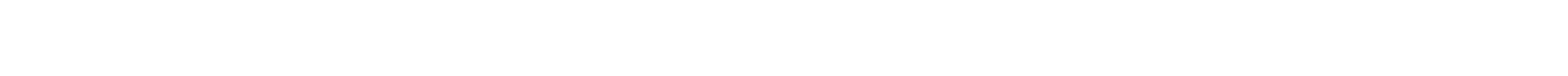

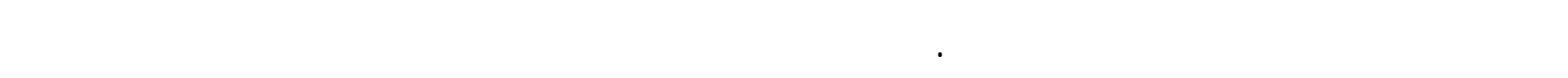

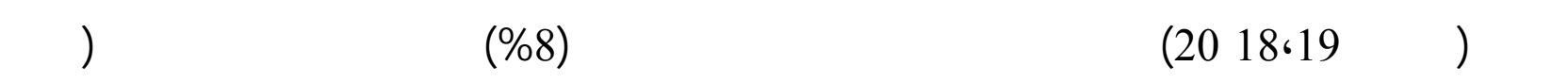

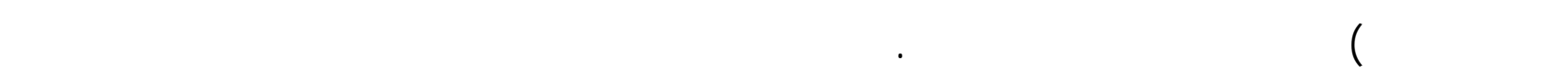

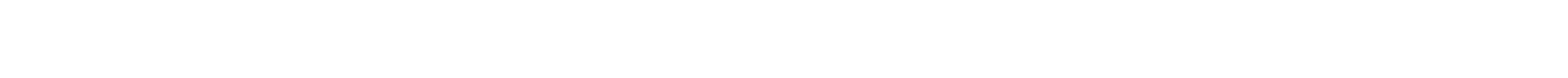

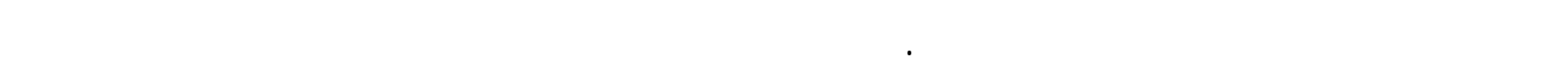
ومتملسكاً، وتتدهور هذه الصفلت عند حصول ظاهيل المرة الفصل الأطوار.

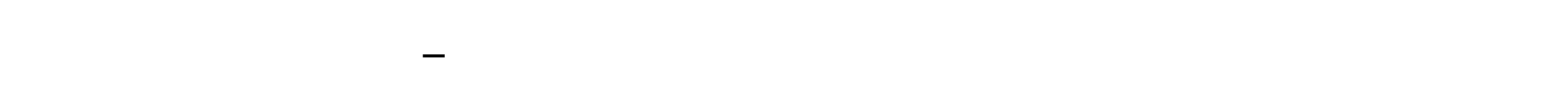

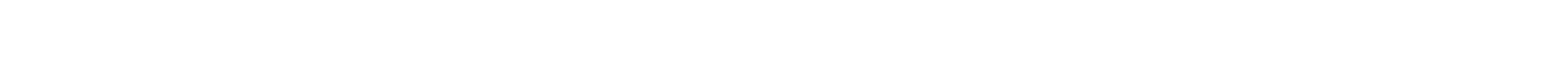

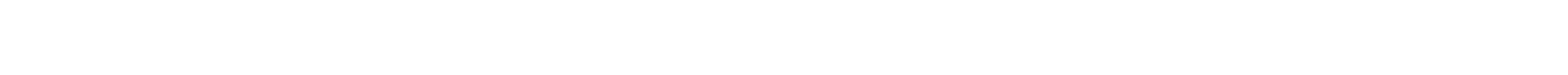

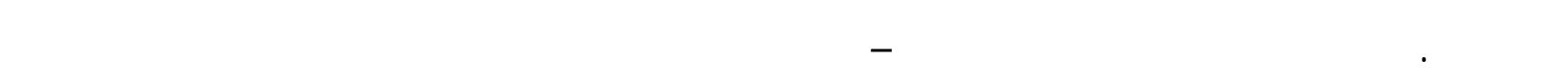

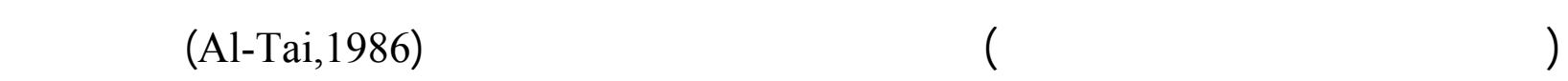

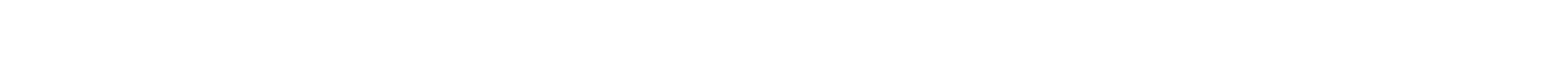

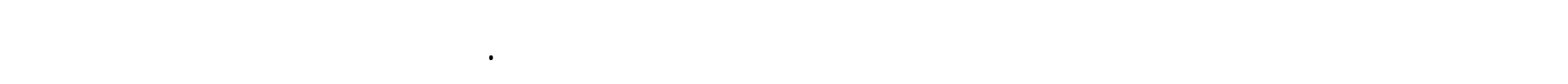

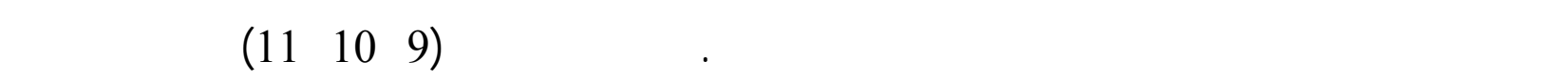

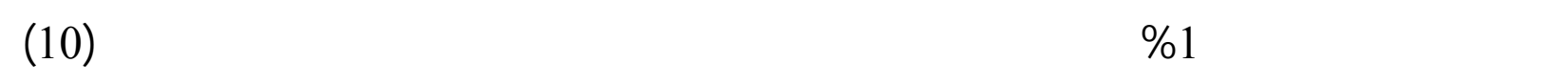
مقارنة بنماذج الأسفلت - كبريت غير الحاوية على بولي أثلين (النماذج 3،2، 4). بـزيادة كمية البولي أثلين

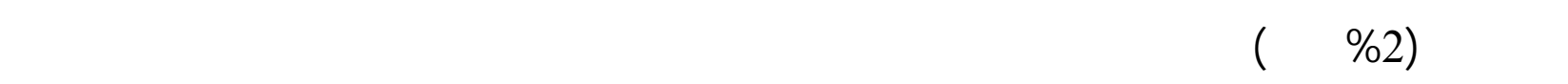

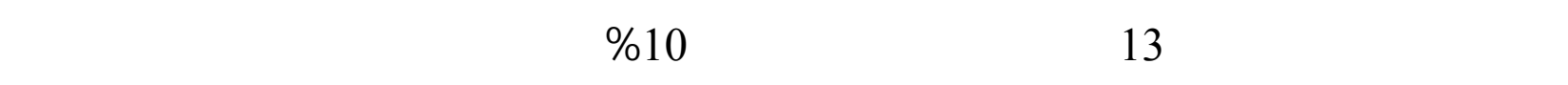

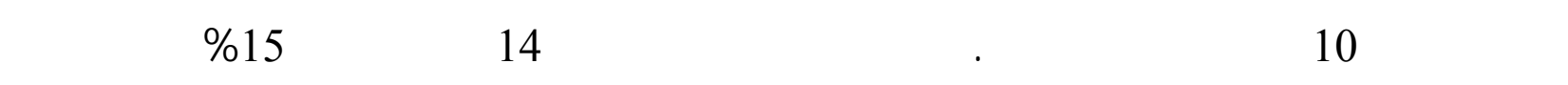

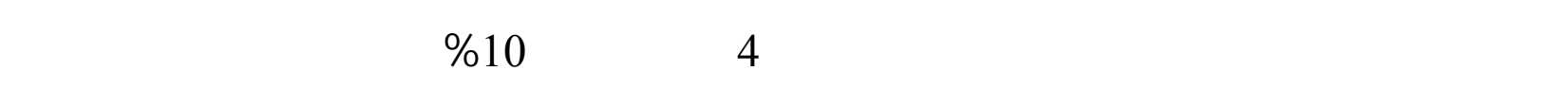
يخط مع البولي أثلين. 


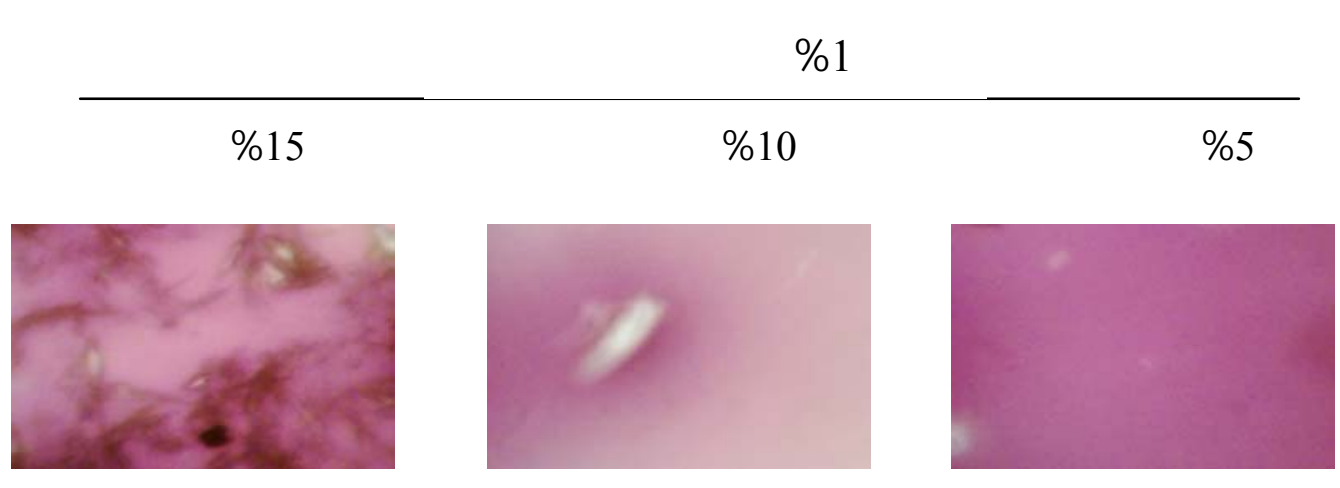

\begin{tabular}{|c|c|c|}
\hline & \% وزنا بولي أثلين & \\
\hline
\end{tabular}
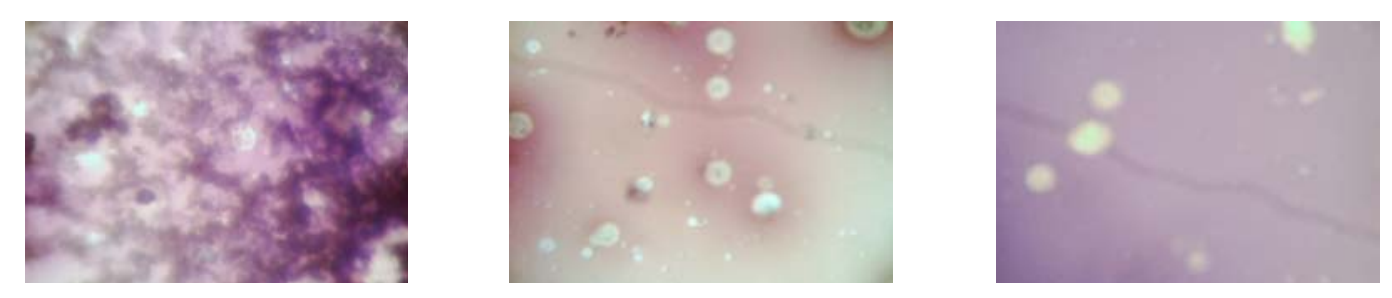

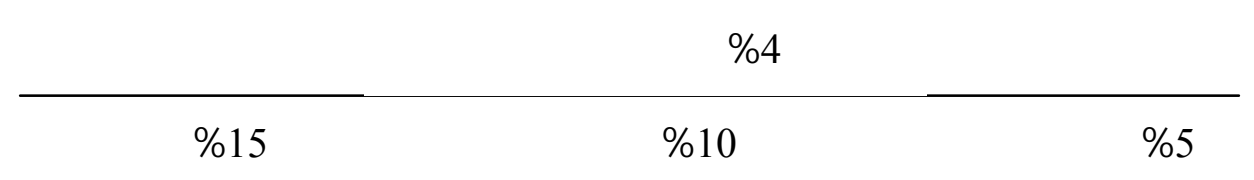
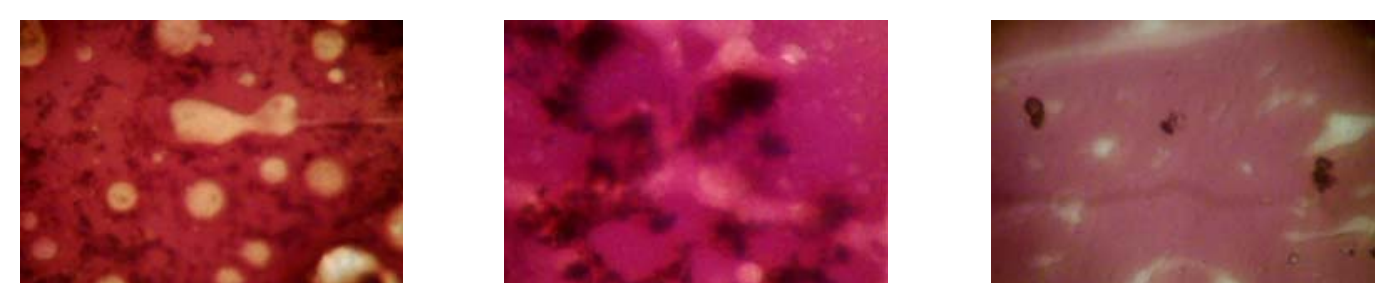

8 وزنا بولي أثلين

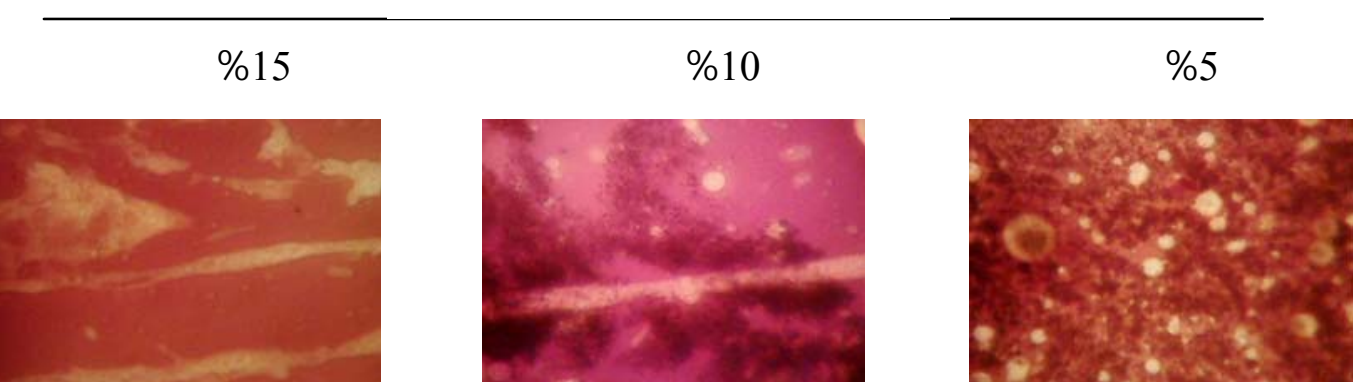

الثكل 2: الصور الميكروسكوبية لثررئح الأشفلت المحور بالبولي أثلين والكبريت معا والمعنقة لأكثرمنسنة. 
عند رفع نسبة البولي أثلين المضافة اله 4\% وزناً (النماذج 15، 16، 17)، نجد أن قابلية الب ـولي

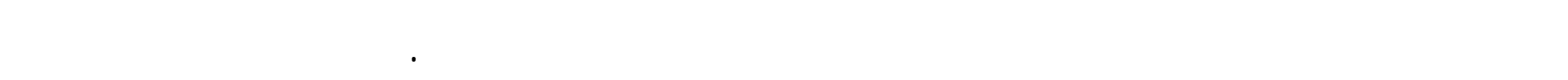

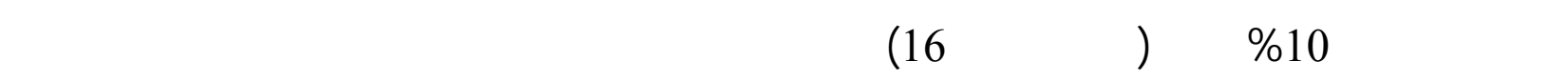

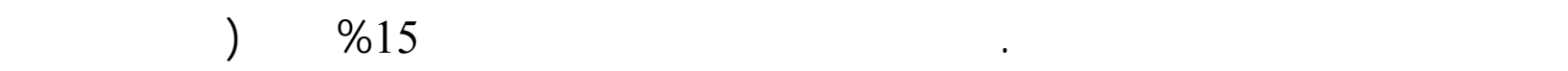

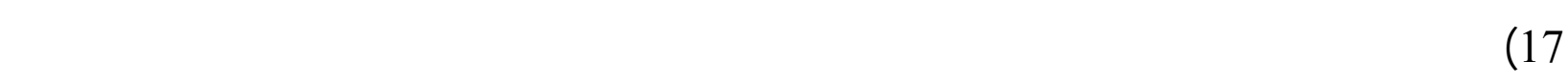
أثلين وبعض المركبلت الأسفلتية القيلة.

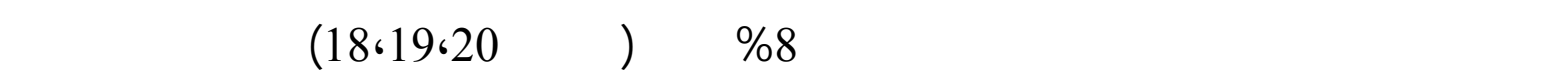

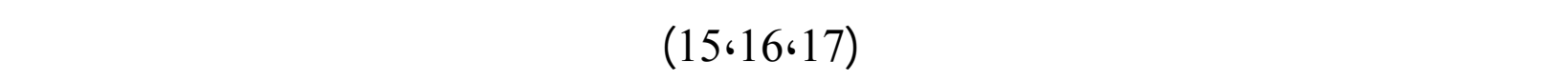

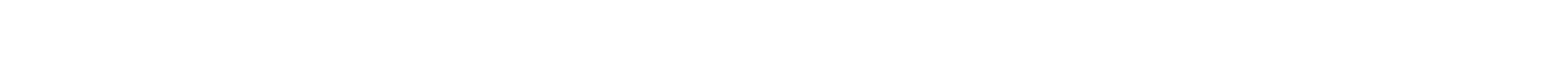

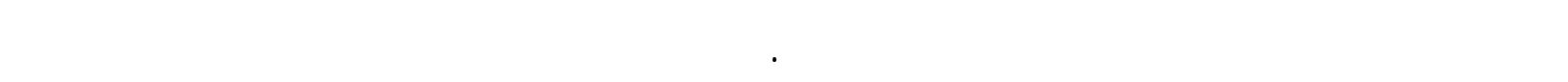

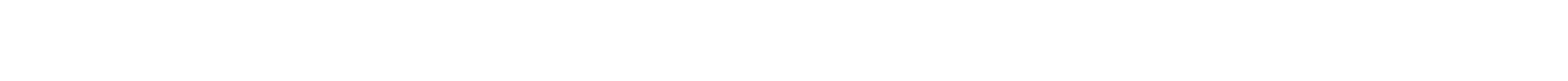

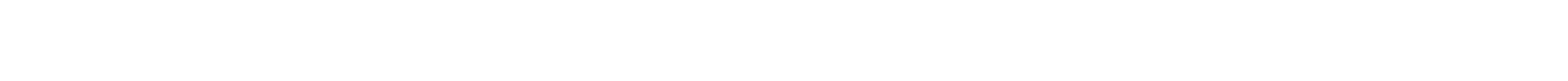
ألجزيئي الأوطأ.

\section{المصادر الاجنبية}

Al-Dobouni, I. A, Abdul Mawjoud A. A., Sofia, G. G. , 2002. Physical and Engineering properties of physically and chemically Sulphur treated Asphalt binders, Engineering \& Technology, Vol.21, No.8.

Al-Dobouni, I. A., Salih, L. A., 2002. Modification of Asphalt by treatment with Polyethylene, Effect of modification methods on the homogeneity of Asphalt, J. Raf. Sci., Vol.13, No.1 related to Chem., pp. 59-66.

Al-Tai, A. K, 1986. Effect of Sulphur on the properties of Asphalt concrete mixtures , M.Sc . Thesis, Eng. College, University of Mosul, pp.55-64.

ASTM, 1972. Part II, (D36-70), 27 p. Standard test method for softening point, ASTM, 1986. Section 4, (D5-83), 97 p.Standard test method for penetration .

ASTM, 1986. Section 4, (D113-83), 127 p. Standard test method for ductility .

Dah-Yinn Lee, 1975. Modification of asphalt and asphalt paving mixtures by Sulphur additives, Ind. Eng. Chem. 14, 171.

Fromm, H. J., Bean, D. C. and Miller. L., 1981. Sulphur-Asphalt pavements performance and recycling, Asphalt Paving Tech ., 50, 98.

Garrigues, G., Nicolau A. and Signouret J. B., 1972. Bitumen preparation containing organic poly-sulfides, Ger. Offten.

Kennepohl, G. J. A. , Logan, A. and Bean, D. C., 1974. Sulphur-Asphalt binders in paving mixtures, Can Sulphur Symp.

L'vov, O., N. and Movchan N., I., 1981. Effect of Sulphur on the properties of roadpaving bitumens and Asphalt- concretes, Visn L'vov politelch. Inst., 46, 155.

Quarles, V. J. J., Ufford, V. and Vlugter, J. C., 1965. Sulphur and bitumen, Brenn Stoff Chem.., 46, 1. 
Rahimian, I. , 1976. Reaction between bitumenand Sulphur and characterization of the resulting compounds, Compend - Dtsch.GesMineraloel - Wiss.Kohlechem. 911, pp. $76-77$.

Samarai, M. A., 1978. Sulphur as a partial substitute in pavement used under hot weather conditions , Canada International Conference on Sulphur in Construction, Canada for Mineral and Energy Technology and Sulphur Development Inst., pp.1215 Sept.

Saylak, D., Gallaway, B. M., 1975.Beneficial use of Sulphur-Asphalt pavements ., J. of Transportation Engineering, J. of ASCE, vol. 13, pp.97 -113.

Sullivan, T. A., McBee, W. C., Rasmussen, K. L. and Kenneth, L., 1975. Sand-SulphurAsphalt paving materials, U.S.Bur.Mines, Rep. Invest. 